\title{
Proteasome-mediated degradation of IKB $\alpha$ and processing of p105 in Crohn disease and ulcerative colitis
}

\author{
Alexander Visekruna, ${ }^{1}$ Thorsten Joeris, ${ }^{1}$ Daniel Seidel, ${ }^{1}$ Anjo Kroesen, ${ }^{2}$ Christoph Loddenkemper, ${ }^{2}$ \\ Martin Zeitz, ${ }^{2}$ Stefan H.E. Kaufmann, ${ }^{1}$ Ruth Schmidt-Ullrich, ${ }^{3}$ and Ulrich Steinhoff' \\ ${ }^{1}$ Max Planck Institute of Infection Biology, Berlin, Germany. ${ }^{2}$ Charité Universitätsmedizin Berlin, Campus Benjamin Franklin, Berlin, Germany. \\ 3Max Delbrück Center of Molecular Medicine, Berlin, Germany.
}

\begin{abstract}
Enhanced NF- $\kappa B$ activity is involved in the pathology of both forms of inflammatory bowel disease (IBD), Crohn disease (CD) and ulcerative colitis (UC). Here we analyzed the mechanism of proteasome-mediated NF- $\kappa B$ activation in CD and UC. Our studies demonstrate that the subunit composition and the proteolytic function of proteasomes differ between UC and CD. High expression of the immunoproteasome subunits $\beta 1 i$ and $\beta 2 i$ is characteristic of the inflamed mucosa of CD. In line with this, we found enhanced processing of $N F-\kappa B$ precursor $\mathrm{p} 105$ and degradation of inhibitor of $N F-\kappa B, I \kappa B \alpha$, by immunoproteasomes isolated from the mucosa of $C D$ patients. In comparison with healthy controls and CD patients, UC patients exhibited an intermediate phenotype regarding the proteasome-mediated processing/degradation of $\mathrm{NF}-\kappa \mathrm{B}$ components. Finally, increased expression of the NF- $\kappa B$ family member $c-$ Rel in the inflamed mucosa of CD patients suggests that $\mathrm{p} 50 / \mathrm{c}$-Rel is important for IFN- $\gamma$-mediated induction of immunoproteasomes via IL-12-driven Th1 responses. These findings suggest that distinct proteasome subunits influence the intensity of NF- $\kappa \mathrm{B}-$ mediated inflammation in IBD patients.
\end{abstract}

\section{Introduction}

Inflammatory bowel disease (IBD) is a term that refers to 2 major chronic intestinal disorders of unknown etiology, Crohn disease (CD) and ulcerative colitis (UC). Genetic and environmental factors, including intestinal microflora, are believed to play a role in the development of IBD (1-4). Although both disease forms share some clinical and pathological features, the mechanisms underlying inflammation differ between CD and UC. While CD is associated with increased production of the Th1 cytokines IL-12, IFN- $\gamma$, and TNF- $\alpha$, UC is characterized by enhanced secretion of the Th2 cytokines IL-5 and IL-13 (5-10).

Aberrant activation of the transcription factor NF- $\mathrm{BB}$ controls the expression of many genes of inflammatory cytokines involved in the pathogenesis of IBD (11-15). In vertebrates, the NF- $\mathrm{B}$

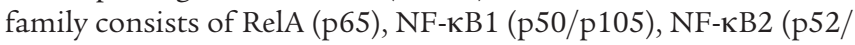
$\mathrm{p} 100$ ), c-Rel, and RelB. In many cell types, the most abundant form of NF- $\mathrm{kB}$ is the $\mathrm{p} 50 / \mathrm{p} 65$ heterodimer, which remains in an inactive state in the cytoplasm, forming a ternary complex with the inhibitory protein inhibitor of NF- $\kappa \mathrm{B}, \mathrm{I} \kappa \mathrm{B} \alpha$. Upon stimulation, I $\mathrm{I} \mathrm{B} \alpha$ is rapidly phosphorylated, ubiquitinated, and subsequently degraded by proteasomes, allowing translocation of p50/p65 heterodimers into the nucleus (16-21). This results in activation of genes related to inflammation and proliferation. Another member of the

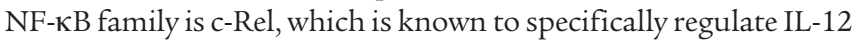
production and consequently Th1 cell differentiation $(22,23)$.

Proteasomes are involved in 2 essential steps of NF- $\mathrm{KB}$ p50/ p 65 activation: generation of $\mathrm{p} 50$ from its precursor $\mathrm{p} 105$ and

Nonstandard abbreviations used: CD, Crohn disease; 2-DE, 2D gel electrophoresis; IBD, inflammatory bowel disease; LCL, lymphoblastoid cell line; MALDI, matrixassisted laser desorption/ionization; MS, mass spectrometry; UC, ulcerative colitis. Conflict of interest: The authors have declared that no conflict of interest exists. Citation for this article: J. Clin. Invest. 116:3195-3203 (2006). doi:10.1172/JCI28804 degradation of IKB $\alpha(24-26)$. In cells, $26 \mathrm{~S}$ proteasomes are responsible for the selective degradation of polyubiquitinated protein substrates. These multicatalytic enzymes consist of two $19 S$ regulatory subunits and the catalytic 20 S core complex, which is composed of 4 heptameric rings (27). Upon stimulation with type I and II interferons, the catalytic constitutive subunits $\beta 1, \beta 2$, and $\beta 5$ are replaced by the inducible $\beta 1 \mathrm{i}$ (LMP2), $\beta 5 \mathrm{i}$ (LMP7), and $\beta 2 \mathrm{i}$ (MECL-1) subunits, leading to the formation of immunoproteasomes, which are characterized by enhanced substrate cleavage (28-32).

Here we isolated proteasomes from intestinal tissues of UC and $\mathrm{CD}$ patients and analyzed their subunit composition as well as their ability to degrade I $\mathrm{I} B \alpha$ and to process p105 in vitro. Our results demonstrate that inflammation induces upregulation of immunoproteasomes in the inflamed mucosa of CD patients and, to a lesser extent, UC patients. Further, we show that processing of p105 and degradation of IкB $\alpha$ is most enhanced by proteasomes derived from CD patients. This correlates with increased amounts of NF-кB in the epithelium and lamina propria of these patients. Finally, our studies suggest that in CD patients, high expression of c-Rel is responsible for immunoproteasome upregulation via IL-12-induced IFN- $\gamma$ secretion.

\section{Results}

Structure and enzymatic activity of 20 S proteasomes in IBD patients. We examined the subunit composition of 20S proteasomes purified from healthy intestinal tissues by 2D gel electrophoresis (2-DE) and subsequently identified proteins by matrix-assisted laser desorption/ionization mass spectrometry (MALDI-MS). More than 70 protein spots representing all 20S proteasome subunits and their isoforms in humans were identified (Figure 1A). We have recently shown that different mouse tissues reveal a characteristic pattern of $20 \mathrm{~S}$ proteasomes (33). We observed that the $\beta 5 \mathrm{i}$ immu- 

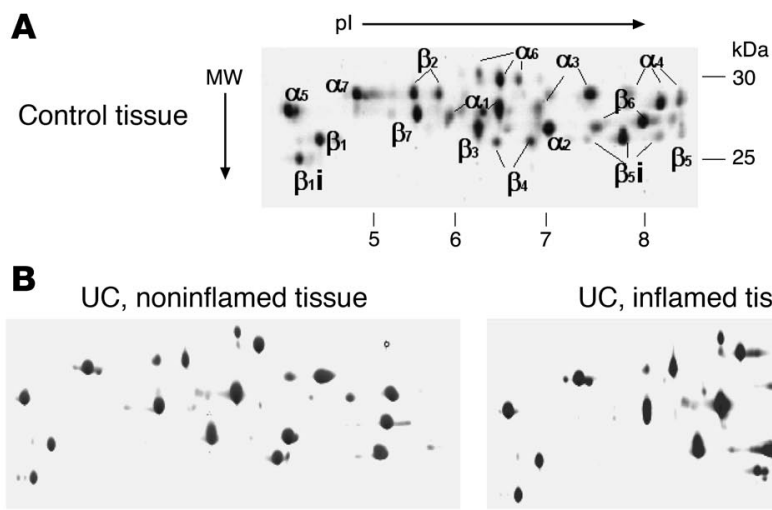

C $\mathrm{CD}$, noninflamed tissue

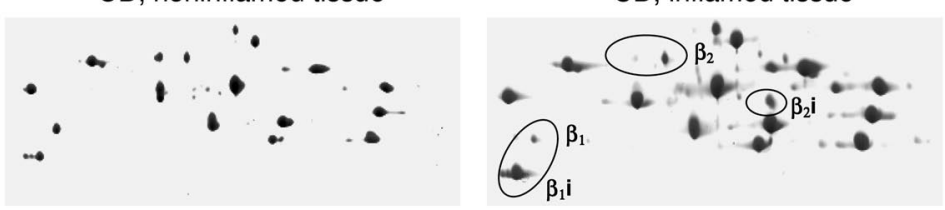

\section{Figure 1}

Subunit composition of proteasomes isolated from the colon of control patients and patients with IBD. Purified 20S proteasomes from the colonic mucosa were separated by 2-DE, and spots were identified by MALDI-MS. (A) 20 S proteasome subunit pattern of control patients $(n=6)$; lines indicate different isoforms of individual subunits. (B and $\mathbf{C}$ ) Subunit composition of 20S proteasomes derived from noninflamed and inflamed mucosa of the colon from UC patients (UC, noninflamed tissue, $n=5$; UC, inflamed tissue, $n=5$ ) and CD patients $(C D$, noninflamed tissue, $n=5 ; C D$, inflamed tissue, $n=5$ ). Circles indicate increased expression of immunosubunits in inflamed mucosa of CD. Shown are representative 2-DE gels of 5 independent experiments. nosubunit was constitutively expressed in the human colon, in contrast with the mouse colon.

Analysis of the impact of inflammation on the structure of $20 \mathrm{~S}$ proteasomes revealed only slight differences in proteasome subunit composition between inflamed and noninflamed colon tissues of UC patients and controls (Figure 1, A and B). In contrast, strong upregulation of the immunosubunits $\beta 1 \mathrm{i}$ and $\beta 2 \mathrm{i}$ was found in the inflamed mucosa of CD patients (Figure 1C and Supplemental Table 1; supplemental material available online with this article; doi:10.1172/JCI28804DS1). To investigate proteolytic activities, we tested 20S proteasomes purified from inflamed colonic mucosa of CD and UC patients and controls for the turnover of fluorogenic substrates, representing trypsin-, chymotrypsin-, and caspase-like activities. CD patients revealed strong chymotrypsin-like activity while UC patients exhibited moderate levels that were marginally enhanced as compared with those of controls. The trypsin-like activity, which is attributed to $\beta 2$ and $\beta 2 \mathrm{i}$ subunits, was similar in all groups. Furthermore, proteasomes of CD patients showed reduced caspase-like activity as compared with UC patients and controls. (Figure 2, A-C). The hydrolytic activity against these substrates strongly depends on the content of immunosubunits. As reported by Groll and Huber (34), the replacement of constitutive subunit $\beta 1$ by the immunosubunit $\beta 1$ results in reduced caspase-like, but enhanced chymotrypsin-like, activity. Consistent with this, the densitometric analysis of Western blots revealed low expression of $\beta 1$ in the mucosa of CD patients compared with UC and control patients (Figure 2, D and E). This finding demonstrates that inflammation strongly affects the proteasome composition in CD but not UC patients.

Induction of immunoproteasomes in the intestinal mucosa and epithelial cells. In order to analyze the distribution of immunoproteasomes, we stained tissue sections of IBD patients for the expression of the immunosubunit $\beta 2 i$. Immunofluorescence studies revealed strong expression of the $\beta 2 \mathrm{i}$ subunit, predominantly in the epithelium and cells of the lamina propria, in the terminal ileum and colon of $C D$ patients. The expression of $\beta 2 \mathrm{i}$ in the inflamed colonic epithelium of UC patients was weaker than in CD patients despite similar histological scores of CD and UC patients. No staining for $\beta 2 \mathrm{i}$ was detectable in tissue sections of the terminal ileum or colon of healthy controls (Figure 3).

To determine signals required for the induction of immunosubunits in epithelial cells, we performed RT-PCR analyses of $\beta 1 \mathrm{i}, \beta 2 \mathrm{i}$, and $\beta 5 \mathrm{i}$ in Caco- 2 cells stimulated with either different amounts of LPS or the inflammatory cytokines IFN- $\gamma$ and TNF- $\alpha$. While stimulation with LPS or TNF- $\alpha$ exhibited no effects, IFN- $\gamma$ stimulation resulted in upregulation of immunosubunits that was not further enhanced by TNF- $\alpha$ (Figure 4). These results confirm previous findings (32) and suggest that immunoproteasome expression in the gut epithelium is induced by IFN- $\gamma$ but not by TNF- $\alpha$ or LPS.

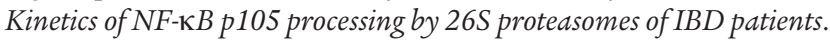
We analyzed the effects of different proteasome subunit composition on processing of the NF- $\mathrm{KB}$ precursor p105 to $\mathrm{p} 50$, which can act either as p50/p65 and p50/c-Rel heterodimers or a p50/ p50 homodimer. Cell lysates from the intestinal mucosa of IBD patients and controls were incubated with in vitro-translated p105 in the presence or absence of ATP. The kinetics of p105 processing demonstrates that the half-life of p105 was approximately 8 minutes and 40 minutes in the presence of proteasomes from CD or UC patients, respectively. After 10 minutes, p105 was almost completely processed by $26 \mathrm{~S}$ proteasomes from $\mathrm{CD}$ patients while proteasomal processing was still incomplete after 120 minutes in UC patients and controls (Figure 5, A and B). Accordingly, the half-life of $\mathrm{p} 105$ in UC patients and controls was increased 5- or 12-fold compared with that of CD patients (Figure $5 \mathrm{D}$ ). In vitro processing of p105 was strictly ATP dependent, demonstrating that generation of p50 from p105 was mediated by $26 \mathrm{~S}$ proteasomes (Figure $5 \mathrm{C}$ ). To exclude effects of contaminating proteases, we performed in vitro processing of p105 in the presence of the proteasome inhibitor MG132 and found complete inhibition by $10 \mu \mathrm{M}$ MG132 (data not shown).

Kinetics of $I \kappa B \alpha$ degradation by $20 S$ proteasomes of IBD patients. Degradation of IKB $\alpha$ is mediated by both $26 \mathrm{~S}$ and $20 \mathrm{~S}$ proteasomes (35). We thus compared the efficiency of $20 \mathrm{~S}$ proteasomes isolated from inflamed mucosa of IBD patients and mucosa of healthy controls in degrading in vitro-translated $\mathrm{I} \kappa \mathrm{B} \alpha$, which is necessary for the activation of the NF-кB p50/p65 complex. 
A
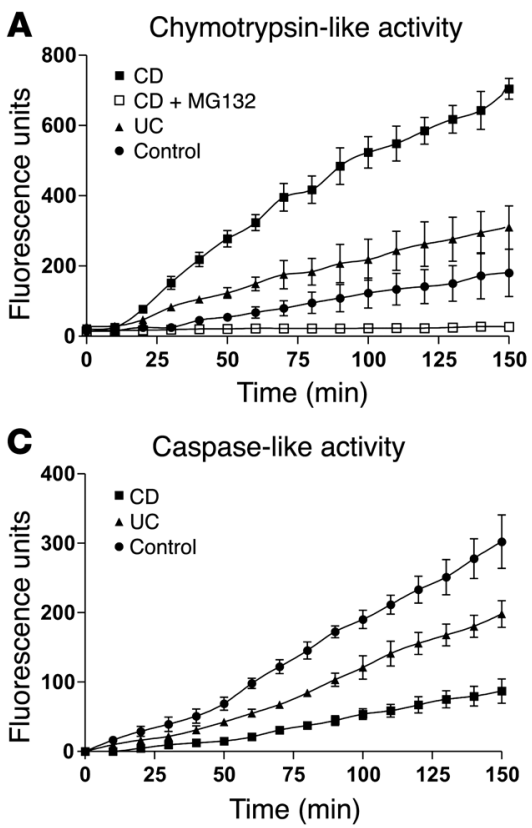

B

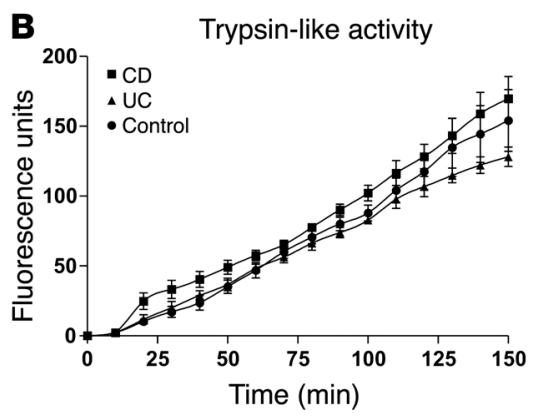

D

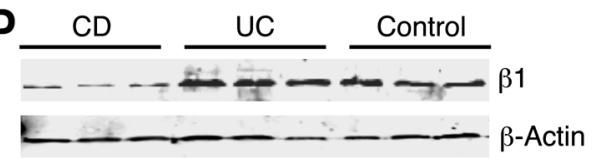

E

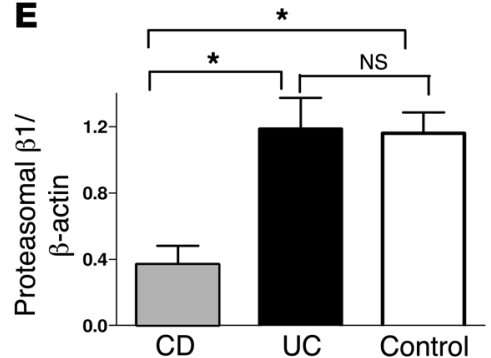

\section{Figure 2}

Proteolytic activity of proteasomes in IBD patients. (A-C) Enzymatic activity of $20 \mathrm{~S}$ proteasomes purified from colonic mucosa of CD patients (filled squares), UC patients (triangles), and control patients (circles) was measured by cleavage of fluorogenic peptide substrates. Control reaction was performed in the presence of the proteasome inhibitor MG132 (open squares). Data are expressed as means \pm SEM. $(n=5)$. Statistical significance was determined by 1-way ANOVA (chymotrypsin- and caspase-like activity, $P<0.001$; trypsin-like activity, $P=0.114)$. (D) Western blot of lysates from inflamed intestinal mucosa of CD patients, UC patients, and controls was stained for the proteasomal immunosubunit $\beta 1$ and $\beta$-actin. (E) Quantitative analysis of the proteasomal $\beta 1 / \beta$-actin protein ratio was performed using AIDA Image Analyser software (version 4.0.3; Raytest). Data represent means \pm SEM; CD $(n=10)$, UC $(n=10)$, and controls $(n=9)$. ${ }^{\star} P<0.05$, Student's $t$ test $(n=9-10)$.
In contrast with very slow proteasomal degradation of $\mathrm{I} \kappa \mathrm{B} \alpha$ in controls, the half-life of IKB $\alpha$ incubated with purified $20 \mathrm{~S}$ proteasomes from CD patients was approximately 7 minutes. Proteasomes from UC patients revealed an intermediate activity,

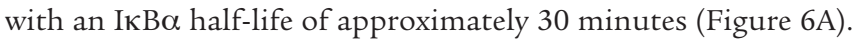
Thus proteasomal degradation of the NF- $\kappa \mathrm{B}$ inhibitory protein IкB $\alpha$ by $C D$ patients was 4 - and 10 -fold faster compared with that of UC patients and controls, respectively (Figure 6B). This finding demonstrates that increased expression of immunosubunits in 20S proteasomes is responsible for the rapid degradation of I $\mathrm{B} \alpha$ observed in $\mathrm{CD}$ patients. To further determine whether the observed effects were due to immunoproteasomes, experiments were repeated with purified $20 \mathrm{~S}$ proteasomes from WT or mutant lymphoblastoid cell lines (LCLs) that either contain (LCL 721) or

\section{Figure 3}

Expression of immunoproteasomes in the intestinal mucosa of IBD patients. (A and $\mathbf{B}$ ) Immunofluorescent staining of sections from the terminal ileum $(\mathbf{A})$ and colon (B) of IBD patients and controls with an antibody to $\beta 2 \mathrm{i}$. Magnification, $\times 200$ (A, upper panel); ×1000 (A, lower panel, and $\mathbf{B})$. (C) Inflammation scores of patients shown in $\mathbf{A}$ and $\mathbf{B}(\mathrm{CD}, n=10$; UC, $n=8$; control, $n=10 ;{ }^{* \star *} P<0.001$ compared with control).
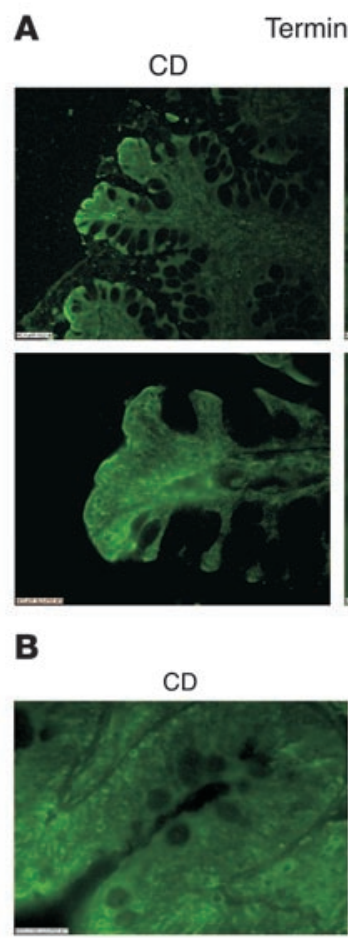

Terminal ileum
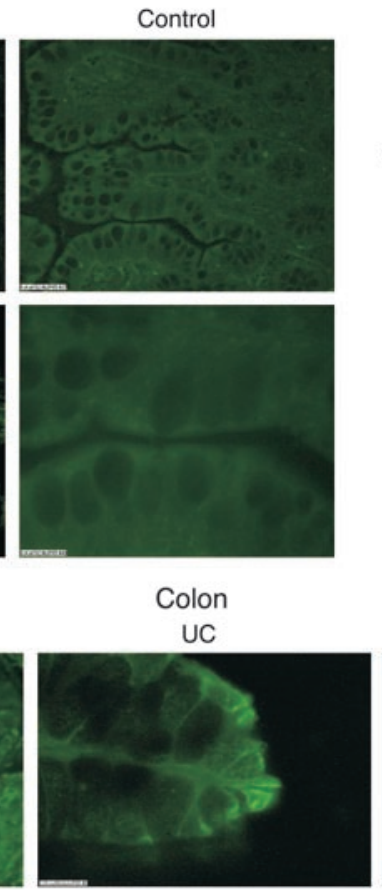
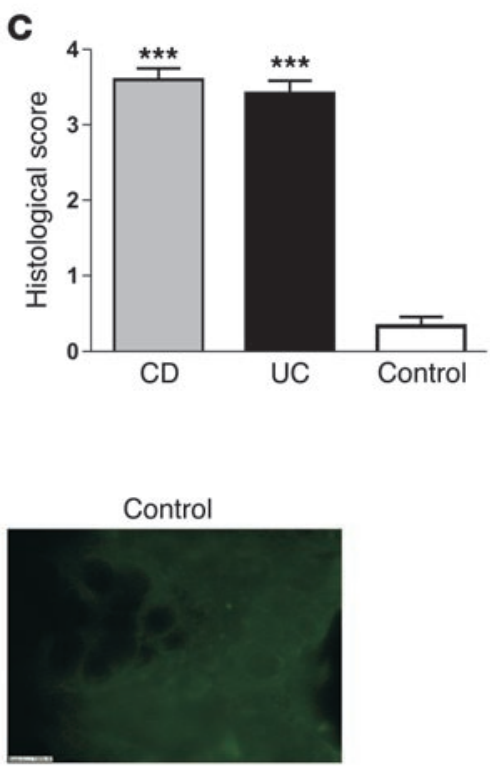
A

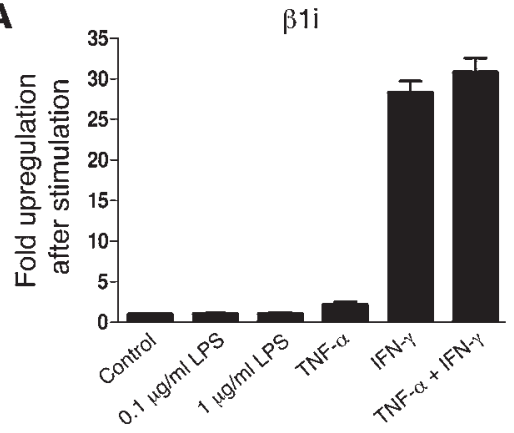

B

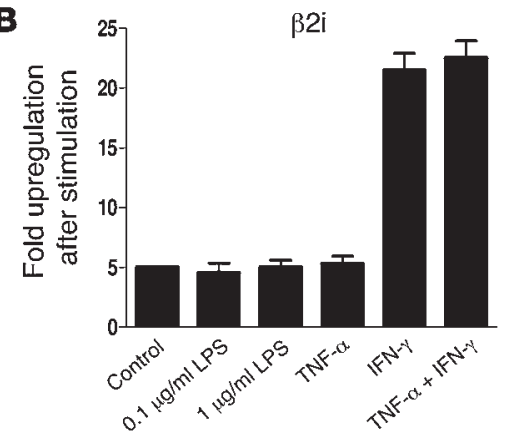

C

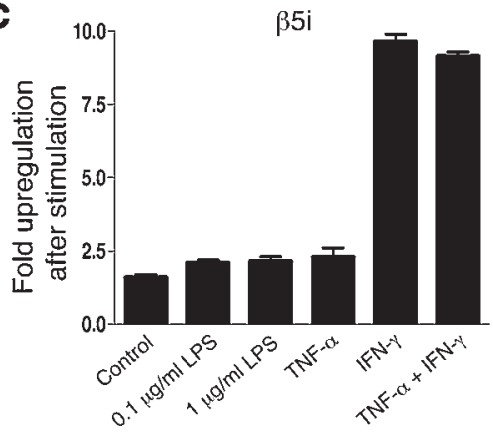

Figure 4

Relative expression of proteasomal immunosubunits in Caco-2 cells. Differentiated Caco-2 cells were stimulated with LPS, TNF- $\alpha$, and IFN- $\gamma$, and the expression of $\beta 1 \mathrm{i}(\mathbf{A})$, $\beta 2 \mathrm{i}(\mathbf{B})$, and $\beta 5 \mathrm{i}(\mathbf{C})$ was measured by real-time PCR. Data represent means $\pm \operatorname{SEM}(n=3)$.

lack immunoproteasomes (LCL 721.174). Whereas 20S proteasomes from LCL 721.174 cells showed high similarity to 20 S proteasomes of control patients, immunoproteasome-rich LCL 721 cells displayed proteolytic activity resembling that of CD patients (Figure 6, C and D, and Supplemental Figure 3).

Effects of a probiotic factor on buman proteasomes and on nascent colitis in mice. Recent data demonstrated that a soluble factor secreted by a mixture of probiotic bacteria (VSL\#3) blocks degradation of $\mathrm{I} \kappa \mathrm{B} \alpha$ and processing of the NF- $\kappa \mathrm{B}$ precursor $\mathrm{p} 105$ by inhibiting the chymotrypsin-like activity of proteasomes in murine cells (36). To examine the effect of this inhibitor on human proteasomes, we preincubated $20 \mathrm{~S}$ proteasomes isolated from inflamed intestines of CD patients with conditioned media from VSL\#3 or E. coli strain Nissle 1917 and performed proteasome activity assays. We observed that the conditioned medium of VSL\#3 but not of E. coli strain Nissle 1917 inhibited the chymotrypsin-like activity of immunoproteasomes from CD patients (Figure 7A). A synthetic proteasome inhibitor MG132 was used as a control. In addition, treatment of IL-10 KO mice with conditioned medium of VSL\#3 before the onset of colitis prevented weight loss during the observation period of 3 weeks as compared with mock-treated mice (Figure 7B). These findings suggest that inhibition of the chymotrypsin-like activity of protea-

\section{Figure 5}

Proteasomal processing of NF-kB precursor protein p105. (A) Kinetics of processing of p105 by proteasomes from the colonic mucosa of IBD patients and controls (CD, $n=6$; UC, $n=5$; controls, $n=5$ ). ${ }^{35} \mathrm{~S}$-labeled in vitro-translated p105 (Supplemental Figure 1) was incubated with cytosolic lysates of colonic tissues in the presence of $10 \mathrm{mM}$ ATP. A representative autoradiogram is shown for each group of patients. (B) Detailed analysis of processing of p105 during first 20 minutes by lysates from CD patients. (C) Processing of p105 in the absence of ATP by lysates from CD patients. (D) Quantitative analysis of autoradiograms, showing significant differences in the processing of p105 among CD patients, UC patients, and controls. Data represent mean values \pm SEM. $P<0.0001$ by 1-way ANOVA. somes with nontoxic bacterial compounds attenuates the development of spontaneous colitis in mice.

Expression of NF- $\kappa B$ components and immunoproteasomes in IBD patients. To determine whether enhanced expression of NF- $\mathrm{\kappa B}$ components is also detectable in the inflamed intestinal mucosa of $\mathrm{CD}$ patients, we compared lysates of CD and UC patients as well as controls for expression of p105 and p50 in the cytosol and the abundance of nuclear p65 and c-Rel. Despite individual variations among patients, we found common patterns for CD and UC patients. Enhanced nuclear expression of NF-KB subunits p65 and c-Rel and very low amounts of $\mathrm{p} 105$ in the cytosol were characteristic for $\mathrm{CD}$ but not for UC patients and controls (Figure 8). These findings support our in vitro data on increased $\mathrm{p} 105$ processing due to immunoproteasomes and suggest that active NF- $\mathrm{BB}$ complexes p50/p65 and p50/c-Rel are predominant in the inflamed mucosa of $\mathrm{CD}$ patients.

Finally, we investigated whether enhanced activation of NF-кB correlates with increased expression of immunoproteasomes in
A

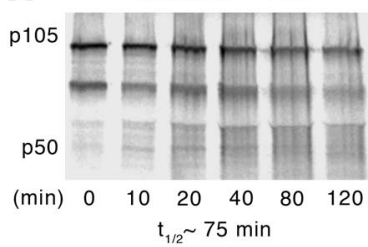

B

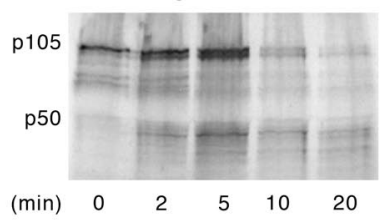

$\mathrm{UC}+\mathrm{ATP}$

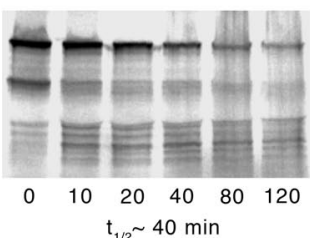

C

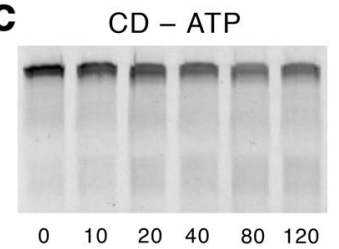

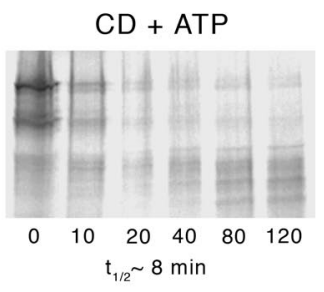

$20 \quad 40$
$t_{1 / 2} \sim 8$

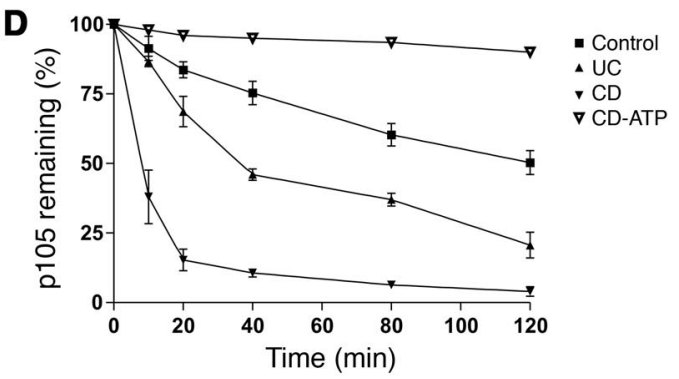


A

Control
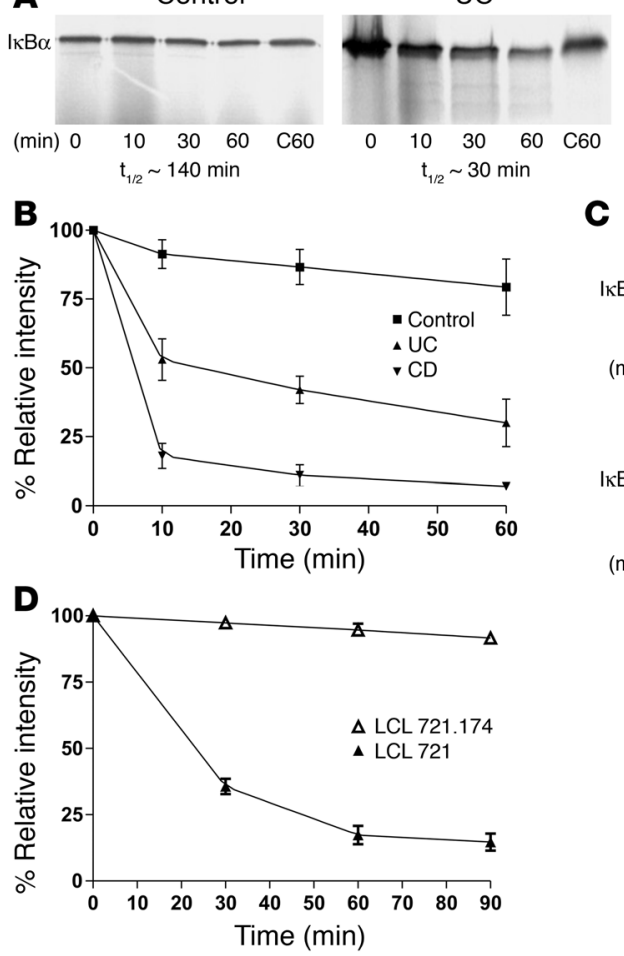

CD

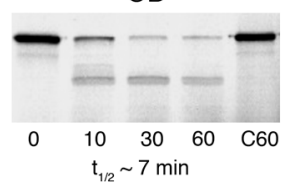

C

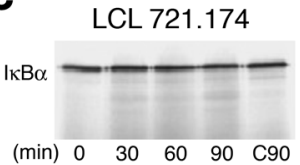

LCL 721

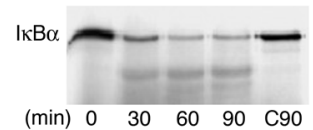

\section{Figure 6}

Effect of immunoproteasomes on degradation of $I_{\kappa} B \alpha$. (A) Kinetics of $I \kappa B \alpha$ degradation by $20 S$ proteasomes purified from colonic mucosa of patients with IBD and controls (CD, $n=5$; UC, $n=5$; controls, $n=5)$. IкB $\alpha$ was in vitro translated in the presence of ${ }^{35}$ S-methionine (Supplemental Figure 2) and incubated with isolated $20 \mathrm{~S}$ proteasomes for indicated times. C60 represents a control incubated for 60 minutes without $20 \mathrm{~S}$ proteasomes with an equivalent amount of $I_{\kappa} B \alpha$. (B) Quantitative evaluation of $I \kappa B \alpha$ degradation by $20 \mathrm{~S}$ proteasomes isolated from patients with IBD and controls in the absence of ATP. $P<0.0001$ (by 1-way ANOVA); $n=5$. (C) Analysis of degradation of in vitro-translated IкB $\alpha$ by 2 different subsets of $20 \mathrm{~S}$ proteasomes. Data from 1 representative experiment are shown for purified constitutive proteasomes (LCL $721.174 ; n=3$ ) and immunoproteasomes (LCL $721 ; n=3$ ). Lack of $\beta 1 \mathrm{i}$ and $\beta 5 \mathrm{i}$ in LCL 721.174 excludes the incorporation of immunosubunits in $20 \mathrm{~S}$ proteasomes. C90 represents a control line without $20 \mathrm{~S}$ proteasomes. (D) Quantification of $\mathrm{I}_{\mathrm{KB}} \alpha$ degradation by $20 \mathrm{~S}$ protesomes isolated from LCL 721 and LCL 721.174 cells $(n=3)$. vivo. We stained control and inflamed tissues of terminal ileum and colon for expression of $\beta 2 \mathrm{i}$ and active NF- $\mathrm{\kappa B}$ p 65 using antibodies specific for the nuclear localization sequence of $\mathrm{p} 65$, which can only be detected after activation of NF-KB. Double immunofluorescence revealed colocalization of activated $\mathrm{p} 65$ and proteasomal immunosubunit $\beta 2 \mathrm{i}$ mainly in the intestinal mucosa of CD patients, suggesting the impact of immunoproteasomes on activation of NF- $\mathrm{BB}$ in vivo (Figure 9). We here propose a mechanism for enhanced NF- $\kappa \mathrm{B}$ activity in CD mediated by immunoproteasomes: Early inflammatory signals trigger expression of c-Rel, which leads to increased IL-12 expression and a strong Th1-mediated $\mathrm{T}$ cell response. High levels of IFN- $\gamma$ induce immunoproteasomes, which are involved in enhanced activation of NF- $\mathrm{KB}$ and exacerbation of inflammation (Figure 10).

\section{Discussion}

In this study, we report that differences in proteasome subunit composition in the inflamed intestines of UC and CD patients have an impact on the level of $\mathrm{NF}-\kappa \mathrm{B}$ activation. Increased processing of 105 and rapid degradation of I $\mathrm{B} \alpha$ by immunoproteasomes in CD patients may be responsible for enhanced expression of inflammatory genes regulated by p50/ c-Rel and p50/p65 heterodimers. Although NF-кB p65 is involved in the pathogenesis of UC and CD, our data show that the abundance and mechanism of induction differ between these disease forms.
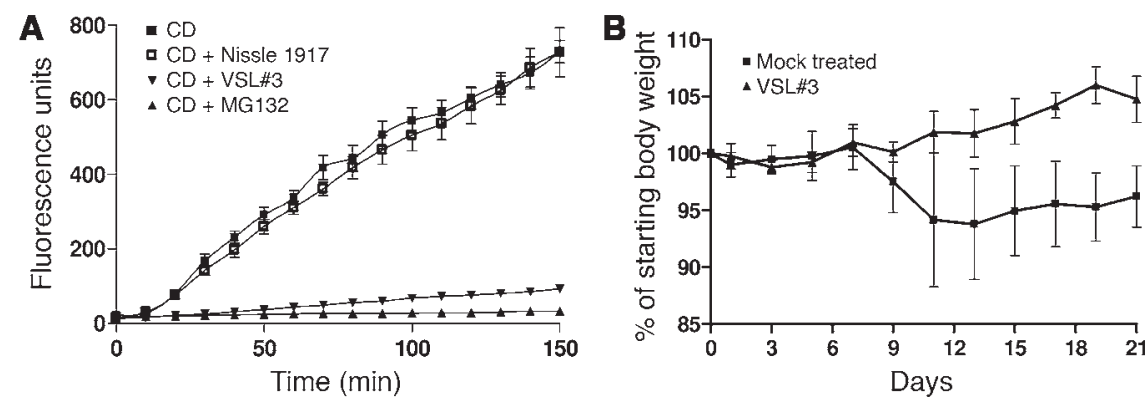

Figure 7

Proteasome inhibitor secreted by probiotic bacteria inhibits the chymotrypsin-like activity of $20 \mathrm{~S}$ proteasomes isolated from CD patients and prevents nascent colitis in IL-10 KO mice. (A) The chymotrypsin-like activity of $20 \mathrm{~S}$ proteasomes $(2 \mu \mathrm{g})$ purified from CD patients $(n=6)$ was measured in the presence $(50 \mu \mathrm{l})$ or absence of conditioned media from probiotic bacteria (VSL\#3) and $E$. coli strain Nissle 1917. As control, the synthetic proteasome inhibitor MG132 was used at a concentration of $10 \mu \mathrm{M}$. (B) IL-10 KO mice were treated before the onset of colitis either with PBS- (control) or VSL\#3-conditioned medium. Treatment started at the age of 10 weeks and was performed for 3 weeks by giving either $500 \mu$ I PBS or VSL\#3 intragastrically every other day. Weight was monitored for both groups $(n=8)$. 


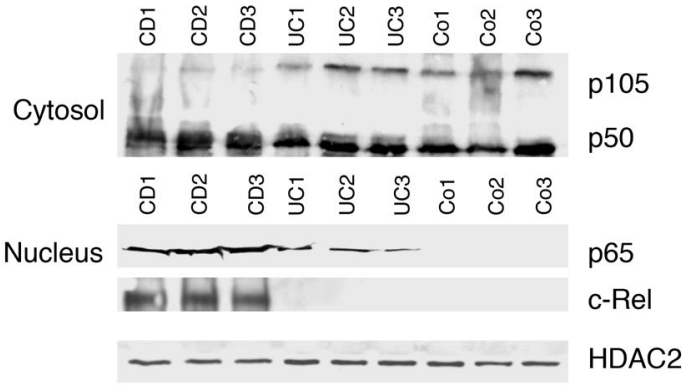

\section{Figure 8}

Cytosolic and nuclear extracts obtained from colonic specimens analyzed by Western blot using antibodies directed against cytosolic p105 and p50 as well as nuclear p65 and c-Rel proteins (CD, $n=9$; UC, $n=9$; controls, $n=10$ ). HDAC2 was used as loading control for nuclear extracts.

somes (37). Incorporation of the IFN- $\gamma$ inducible subunits $\beta 1 \mathrm{i}$ and $\beta 2 \mathrm{i}$ in proteasomes of CD patients altered their proteolytic activity. While trypsin-like activity remained unchanged, immunoproteasomes of CD patients revealed enhanced chymotrypsin-like and reduced caspase-like activity as compared with UC patients and controls. However, it must be mentioned that, compared with controls, increased chymotrypsin- and decreased caspase-like activities were also found in UC, although the differences were less prominent than in $\mathrm{CD}$ patients.

We obtained evidence for the involvement of immunoproteasomes in enhanced NF-KB activation when we showed that in vitro processing of NF- $\kappa \mathrm{B}$ p105 and degradation of $\mathrm{I} \kappa \mathrm{B} \alpha$ were accelerated in $\mathrm{CD}$ patients. Compared with healthy controls and UC patients, proteasomal processing of p105 in the inflamed colonic mucosa of CD patients was 12- or 5-fold faster, respectively. Kinetic studies further demonstrated that the halflives of I $\mathrm{B} \alpha$ degraded by purified $20 \mathrm{~S}$ proteasomes from CD patients was shortest (7 minutes), followed by those of UC patients (30 minutes), and was longest in controls (140 minutes). Together with the data from cell lines demonstrating that degradation of $\mathrm{I \kappa B} \alpha$ is directly linked to the expression of proteasomal immunosubunits, these results confirm the profound effect of different proteasome subsets on NF- $\mathrm{KB}$ activation. Thus the hierarchy of immunosubunit expression in IBD patients is reflected by their function.

An explanation for high immunosubunit expression in $\mathrm{CD}$ patients may be provided by the strong expression of c-Rel in the inflamed mucosa of CD patients. Increased amounts of p50 and c-Rel suggest formation

\section{Figure 9}

Double immunofluorescence of proteasomal immunosubunit $\beta 2 i$ and NF- $\mathrm{KB}$ subunit $\mathrm{p} 65$ in the terminal ileum and colon of IBD patients and controls. Magnification, $\times 1,000$. Increased expression of NF- $\mathrm{KB}$ subunit $\mathrm{p} 65$ colocalizes with enhanced expression of the $\beta 2 \mathrm{i}$ subunit in intestinal epithelial cells and cells of the lamina propria (green fluorescence, $\beta_{2}$ i; red fluorescence, p65). NF-кB was stained with a primary antibody that exclusively recognizes activated p65. Data represent 1 of 8-10 patients per group. of p50/c-Rel complexes, which were previously shown to regulate IL-12 expression $(22,23)$. IL-12 is a key cytokine for Th1 cell polarization. This in turn initiates IFN- $\gamma$ production and thus controls the induction of immunoproteasomes. The distinction of UC and $\mathrm{CD}$ patients with respect to $\mathrm{c}$-Rel expression in the inflamed mucosa confirms previous observations that $\mathrm{CD}$ is associated with a Th1 cell response whereas UC exhibits more of a Th2-like cytokine pattern $(1,5)$. In accordance with this, treatment of Th1-mediated colitis in animals and CD patients with anti-IL-12 and anti-TNF- $\alpha$ antibodies has been shown to improve inflammatory reactions $(38,39)$.

The involvement of proteasomes in intestinal inflammation has also been emphasized by studies from Montelone et al. (40, 41). In addition to the direct activation of NF- $\kappa \mathrm{B}$, proteasomes also control inflammation indirectly by degrading Smad7, an inhibitor of TGF- $\beta 1$ signaling. In normal tissues, TGF- $\beta 1$ signaling nega-

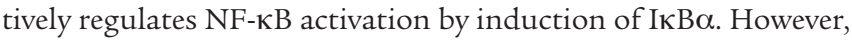
in the inflamed gut of IBD patients, high expression of Smad7, an intracellular inhibitor of TGF- $\beta 1$ signaling, allows inflammation to proceed unchecked. Posttranscriptional acetylation of Smad7 in IBD patients is believed to be responsible for its resistance to proteasomal degradation that normally occurs in the healthy gut.

Many of the antiinflammatory drugs used to treat experimental colitis are based on the inhibition of NF- $\mathrm{\kappa B}$ activation, which underlines its importance as a pathogenic factor. Blocking NF-кB activity with antisense oligonucleotides specific for $\mathrm{p} 65$ and decoy oligonucleotides targeting the NF- $\mathrm{KB}$ consensus binding sequence has been shown to be a potent method to terminate or even reverse established inflammation $(11,42)$. As this might be the most
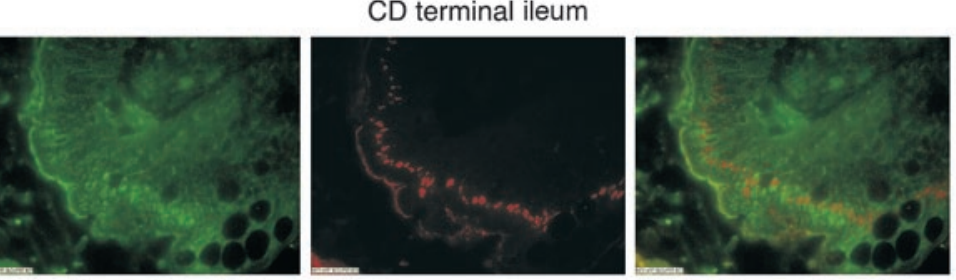

CD colon
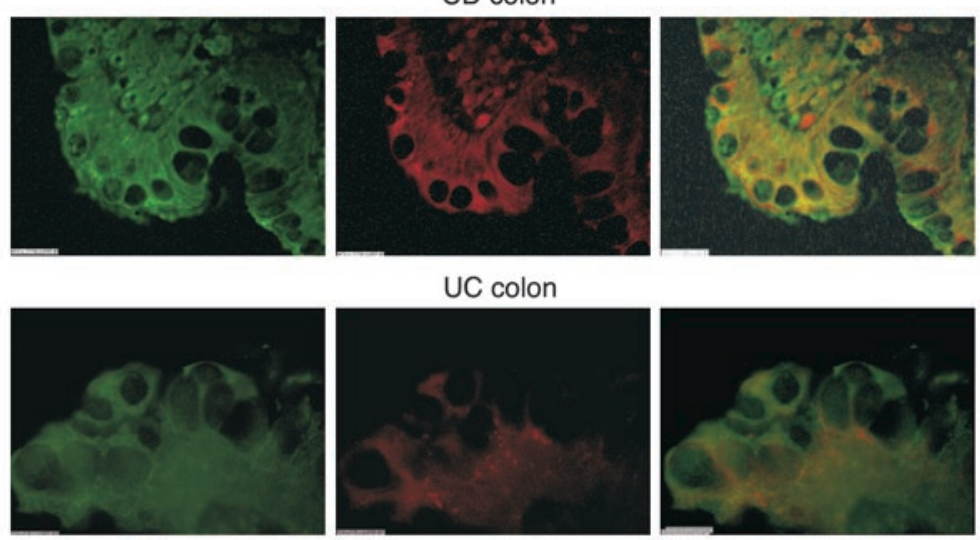

Control colon
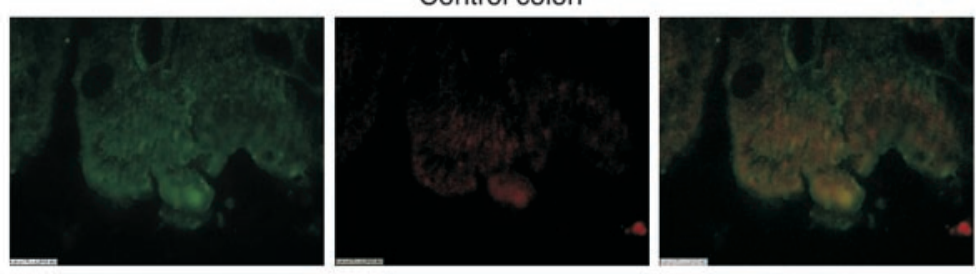


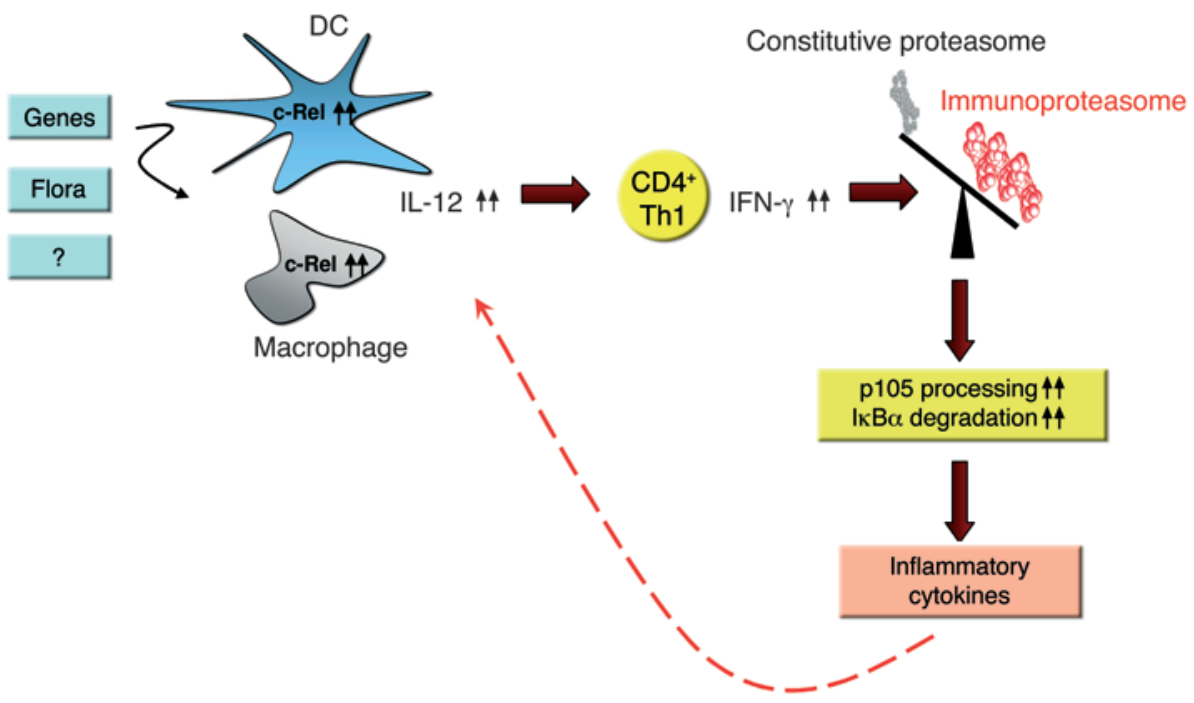

\section{Figure 10}

Schematic overview of enhanced immunoproteasome-mediated NF- $\mathrm{KB}$ activation and consequent exacerbation of inflammation in CD patients. Unknown initial inflammatory events induce expression of $\mathrm{c}$-Rel in the intestinal mucosa and a strong Th1 cell response via IL-12. High amounts of IFN- $\gamma$ trigger generation of immunoproteasomes, which enhances $\mathrm{NF}-\kappa \mathrm{B}$ activation, thus perpetuating the inflammatory process. effective method to rapidly treat established IBD, it remains to be determined whether a complete blockage of major components of NF-кB is also suitable for long-term treatment. Since controlled inflammation represents a crucial defense mechanism against various pathogens, complete blockade of the inflammatory response could strongly affect antimicrobial resistance in these patients. In this study, we show that a proteasome inhibitor secreted by probiotic bacteria specifically inhibits the chymotrypsin-like activity of $20 \mathrm{~S}$ proteasomes from CD patients and attenuates the development of spontaneous colitis in IL-10 KO mice. However, it remains to be determined whether specific inhibition of immunosubunits $\beta 1 \mathrm{i}$ and $\beta 2 \mathrm{i}$ can block excessive NF- $\mathrm{\kappa B}$ activation without ablating the NF- $\mathrm{KB}-$ mediated inflammatory reactions required for protection against pathogens.

\section{Methods}

Patients. Human tissue was obtained from colonic and small intestinal specimens immediately after intestinal surgery performed at the Charité University Hospital in Berlin. All patients provided informed consent. This study was approved by the local ethics committee of the Charite Universitätsmedizin Berlin. A total of 25 patients with active CD, 20 patients with active UC, and 28 tumor patients acting as controls were investigated. In 9 CD patients, the primary site of disease was the colon, and in 16 patients, both terminal ileum and colon were involved. At the time of sample collection, 15 patients of the $\mathrm{CD}$ group were receiving corticosteroids, 4 were receiving azathioprine, 3 were receiving 5-ASA, and 4 were on no medications. In the UC group, 11 patients were receiving corticosteroids, 4 were receiving azathioprine, and 6 were not treated with any immunosuppressive drug. Mucosal tissue layers were mechanically dissected from the underlying submucosa, immediately frozen in liquid nitrogen, and stored at $-196^{\circ} \mathrm{C}$ until use. The degree of inflammation in the surgical specimens was evaluated using a scoring system for UC as follows: 0 , no inflammation; 1 , mild, chronic, inactive inflammation (architectural changes, chronic inflammatory infiltrate); 2 , mild, active inflammation (lamina propria neutrophils); 3 , moderate, active inflammation (crypt destruction); 4, severe, active (erosions or ulcerations) inflammation. The scoring system for $\mathrm{CD}$ was similar: 0 , normal; 1 , mild edema and inflammation in the lamina propria; 2 , crypt abscesses and moderate lamina propria inflammation; 3 , more severe inflammation with destructive crypt abscesses plus or minus granulomata; 4, fulminant inflammation with ulcerations or fissures.
Mice. C57BL/6 mice and IL10-/- mice on C57BL/6 background were bred in the animal facilities of the Max Planck Institute for Infection Biology under specific pathogen-free conditions. Experiments using animals were approved by the local ethics committee of the Charite Universitätsmedizin Berlin and by the German authorities (Landesamtes für Gesundheit und Soziales Berlin).

Cell lines. LCL 721 and LCL 721.174 cells were described previously (43). Cell lines were provided by V. Cerundolo (John Radcliffe Hospital, Oxford, United Kingdom). Caco-2 cells were purchased from ATCC.

Preparation of bacteria-conditioned media. Probiotic bacterial mixture VSL\#3 (VSL Pharmaceuticals) and E. coli strain Nissle 1917 were grown to a concentration of approximately $10^{14}$ bacteria in phenol red-free RPMI 1640 medium (Invitrogen) for 16 hours and then centrifuged. Supernatants were passed through a $0.22-\mu \mathrm{m}$ filter and used in proteasome activity assays $(50 \mu \mathrm{l})$ and oral treatment of mice $(500 \mu \mathrm{l})$.

Purification of 20 S proteasomes. The $20 \mathrm{~S}$ proteasomes were purified as previously described (33). For preparation of proteasomes from the small intestine, the 3-fold higher concentration of protease inhibitors was used. The purity of $20 \mathrm{~S}$ proteasome preparations was tested by SDS-PAGE.

2-DE and MALDI. The $20 \mathrm{~S}$ proteasome preparations were desalted and concentrated by trichloracetic acid precipitation. Pellets were resolubilized in 2-DE sample buffer and subjected to electrophoresis. For resolution of 20 proteasomal subunits, isoelectric focusing by carrier ampholytes was combined with SDS-PAGE. Between 20 and $80 \mu$ g proteins were applied to the anodic side of a carrier ampholyte isoelectric focusing gel. In the second dimension, the proteins were separated in SDS-PAGE gels $(23 \times 30 \mathrm{~cm}$ and $7 \times 8 \mathrm{~cm}$ ) and stained by Coomassie Brilliant Blue G250 and silver, respectively. For mass spectrometric identification, each individual spot detected on the 2D gel was cut out, digested by trypsin, desalted and concentrated by ZipTip (Millipore), and analyzed by MALDI-MS (4700 Proteomics Analyzer; Applied Biosystems). Finally, database searches were performed using combined peptide mass fingerprinting and MS/MS datasets.

Proteasome activity assay. Proteolytic activities of proteasomes were assayed with fluorogenic peptide substrates. Purified $20 \mathrm{~S}$ proteasomes $(1 \mu \mathrm{g})$ were added to the reaction buffer $(50 \mathrm{mM}$ Tris, $\mathrm{pH} 7.5,10 \mathrm{mM} \mathrm{NaCl}, 30 \mathrm{mM}$ $\mathrm{KCl}, 0.1 \mathrm{mM}$ EDTA, and $20 \mu \mathrm{M}$ substrate), and fluorescence was detected by fluorescence microtiter reader Fluoroskan Ascent (Thermo Labsystems; Thermo Electron Corp.) at 355-nm excitation/460-nm emission for 7-amino-4-methylcoumarin (AMC). Caspase-like activity was assayed with Z-Leu-Leu-Glu-AMC, chymotrypsin-like activity with Suc-Leu-Leu- 
Val-Tyr-AMC, and trypsin-like activity with Bz-Val-Gly-Arg-AMC as substrates. Inhibitor studies were carried out by assaying proteolytic activities of the proteasomes after preincubation with MG132 at a concentration of $10 \mu \mathrm{M}$. For kinetic assays, fluorescence was measured every 10 minutes and data were analyzed using Ascent software, version 2.6 (Thermo Labsystems; Thermo Electron Corp.). All experiments were performed at least 3 times.

Western blot analysis. Proteasomal subunit $\beta 1$ was detected using rabbit anti-human antibody (Affinity Research Products). Nuclear NF-KB subunit $\mathrm{p} 65$ was stained with a rabbit anti-human NF-кB/p65 antibody (Santa Cruz Biotechnology Inc.) and cytosolic p50 and its precursor p105 with a rabbit anti-human NF-кB/p50 and p105 antibody (eBioscience). The specific antibody against NF- $\mathrm{KB}$ subunit c-Rel was purchased from Rockland Immunochemicals. As control for protein amounts, a monoclonal antihuman $\beta$-actin antibody (Sigma-Aldrich) and a rabbit anti-human histone deacetylase (HDAC2) antibody (Sigma-Aldrich) were used.

RT-PCR. Total RNA from differentiated Caco- 2 cells was extracted with TRIzol (Invitrogen) and treated with RNase-free DNase to remove contaminating genomic DNA from the sample. RNA quality was checked with the 2100 Bioanalyzer (Agilent Technologies). After reverse transcription, real time-PCR was performed with $2 \times$ SYBR Green PCR Master Mix (Applied Biosystems) using an ABI Prism 7000 Sequence Detection System (Applied Biosystems).

Immunofluorescence studies. Immunofluorescence studies were performed using intestinal cryosections. Normal goat serum was applied to block nonspecific protein binding. As primary antibodies, a polyclonal rabbit antibody against human $\beta 2 \mathrm{i}$ (Affinity Research Products) and a monoclonal mouse antibody against NF- $\mathrm{KB}$ p 65 subunit (Chemicon International) were used. This was followed by incubation with secondary IgG antibody conjugated with Cy 2 and $\mathrm{Cy} 3$, respectively.

Preparation of cytosolic and nuclear extracts from intestinal tissue. Ground intestinal tissue was immersed in ice-cold lysis buffer (10 mM HEPES, pH 7.9, $10 \mathrm{mM} \mathrm{KCl}, 1.5 \mathrm{mM} \mathrm{MgCl}_{2}, 1 \mathrm{mM}$ DTT, $1.5 \mathrm{mM}$ PMSF, $20 \mathrm{mM} \mathrm{NaF}$, $200 \mu \mathrm{M} \mathrm{Na}_{3} \mathrm{VO}_{4}$, and protease inhibitor cocktail). After keeping the sample on ice for 15 minutes, $25 \mu \mathrm{l} 10 \% \mathrm{NP}-40$ was added per $1 \mathrm{ml}$ homogenate. Further samples were incubated on ice for another 20 minutes and centrifuged at $10,000 \mathrm{~g}$ for 2 minutes at $4^{\circ} \mathrm{C}$. Supernatants were used as cytosolic extracts. Pelleted nuclei were washed with PBS and resuspended in extraction buffer (20 mM HEPES, pH 7.9, $420 \mathrm{mM} \mathrm{NaCl}, 1.5 \mathrm{mM} \mathrm{MgCl}_{2}, 0.2$ mM EDTA, 1 mM DTT, $1.5 \mathrm{mM}$ PMSF, $20 \mathrm{mM} \mathrm{NaF}, 200 \mu \mathrm{M} \mathrm{Na}_{3} \mathrm{VO}_{4}$, and protease inhibitor cocktail). Finally, nuclear suspensions were centrifuged at $10,000 \mathrm{~g}$ for 30 minutes at $4^{\circ} \mathrm{C}$ to collect the supernatants containing nuclear protein extracts.

In vitro processing of $p 105$ and $I \kappa B \alpha$ degradation assay. Coding sequences of $\mathrm{p} 105$ and ІкB $\alpha$ were cloned into the expression vector pcDNA3 harboring FLAG epitope, respectively. These constructs were subjected to a reticulocyte lysate-based coupled transcription/translation in vitro system (Promega) in the presence of ${ }^{35} \mathrm{~S}$-methionine. Afterward, ${ }^{35} \mathrm{~S}$-labeled p105 and I $\mathrm{B} \alpha$ proteins were used as substrates for in vitro processing/degradation assay. The ${ }^{35}$ S-labeled $\mathrm{p} 105$ was incubated with cell lysates $(80 \mu \mathrm{g}$ of

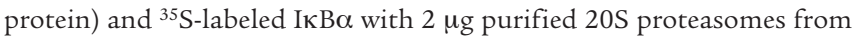
the intestinal mucosa of IBD patients and controls, respectively. The processing/degradation was measured at $37^{\circ} \mathrm{C}$ in the absence or presence of $10 \mathrm{mM}$ ATP. Control reactions contained $10 \mu \mathrm{M}$ MG132 (Sigma-Aldrich). The samples were quantified using LAS-3000 imaging system (Fujifilm).

Statistics. Data were analyzed using 2-tailed Student's $t$ test. For analyzing multiple groups, 1-way ANOVA was used, and results were considered significant at $P \leq 0.05$.

\section{Acknowledgments}

This study was supported by Sonderforschungsbereich 633 (to U. Steinhoff and A. Kroesen) and SFB 421 (to U. Steinhoff). We thank P. Krienke, U. Zimny-Arndt, D. Oberbeck-Mueller, N. Slavova, S. Dullat, and M. Schmid for technical support and U. Kuckelkorn and P. Kloetzel for helpful discussions.

Received for publication April 12, 2006, and accepted in revised form October 3, 2006.

Address correspondence to: Ulrich Steinhoff, Max Planck Institute of Infection Biology, Schumannstrasse 20-21, 10117 Berlin, Germany. Phone: 49-30-28460-560; Fax: 49-30-28460-503; E-mail: steinhoff@mpiib-berlin.mpg.de.
1. Bouma, G., and Strober, W. 2003. The immunological and genetic basis of inflammatory bowel disease. Nat. Rev. Immunol. 3:521-533.

2. Sartor, R.B. 1997. The influence of normal microbial flora on the development of chronic mucosal inflammation. Res. Immunol. 148:567-576.

3. Podolsky, D.K. 2002. Inflammatory bowel disease. N. Engl. J. Med. 347:417-429.

4. Ogura, Y., et al. 2001. A frameshift mutation in NOD2 associated with susceptibility to Crohn's disease. Nature. 411:603-606.

5. Neurath, M.F., Finotto, S., and Glimcher, L.H. 2002. The role of Th1/Th2 polarization in mucosal immunity. Nat. Med. 8:567-573.

6. Monteleone, G., et al. 1997. Interleukin 12 is expressed and actively released by Crohn's disease intestinal lamina propria mononuclear cells. Gastroenterology. 112:1169-1178.

7. Parronchi, P., et al. 1997. Type 1 T-helper cell predominance and interleukin-12 expression in the gut of patients with Crohn's disease. Am. J. Pathol. 150:823-832

8. Murch, S.H., Braegger, C.P., Walker-Smith, J.A., and Macdonald, T.T. 1993. Location of tumour necrosis factor alpha by immunohistochemistry in chronic inflammatory bowel disease. Gut. 34:1705-1709.

9. Fuss, I.J., et al. 1996. Disparate CD4+ lamina propria (LP) lymphokine secretion profiles in inflammatory bowel disease. Crohn's disease LP cells man- ifest increased secretion of IFN-gamma, whereas ulcerative colitis LP cells manifest increased secretion of IL-5. J. Immunol. 157:1261-1270.

10. Heller, F., et al. 2005. Interleukin-13 is the key effector Th2 cytokine in ulcerative colitis that affects epithelial tight junctions, apoptosis, and cell restitution. Gastroenterology. 129:550-564.

11. Neurath, M.F., Pettersson, S., Meyer Zum Buschenfelde, K.H., and Strober, W. 1996. Local administration of antisense phosphorothioate oligonucleotides to the p 65 subunit of NF-kappa B abrogates established experimental colitis in mice. Nat. Med. 2:998-1004.

12. Neurath, M.F., and Pettersson, S. 1997. Predominant role of NF-kappa B p65 in the pathogenesis of chronic intestinal inflammation. Immunobiology. 198:91-98.

13. Barnes, P.J., and Karin, M. 1997. Nuclear factor-kappaB: a pivotal transcription factor in chronic inflammatory diseases. N. Engl. J. Med. 336:1066-1071.

14. Rogler, G., et al. 1998. Nuclear factor kappaB is activated in macrophages and epithelial cells of inflamed intestinal mucosa. Gastroenterology. 115:357-369.

15. Schreiber, S., Nikolaus, S., and Hampe, J. 1998. Activation of nuclear factor kappa B inflammatory bowel disease. Gut. 42:477-484.

16. Ghosh, S., and Baltimore, D. 1990. Activation in vitro of NF-kappa B by phosphorylation of its inhibitor I kappa B. Nature. 344:678-682.
17. Henkel, T., et al. 1993. Rapid proteolysis of I kappa $\mathrm{B}$-alpha is necessary for activation of transcription factor NF-kappa B. Nature. 365:182-185.

18. Karin, M., and Ben Neriah, Y. 2000. Phosphorylation meets ubiquitination: the control of NF-kappaB activity. Annu. Rev. Immunol. 18:621-663.

19. Ghosh, S., and Karin, M. 2002. Missing pieces in the NF-kappaB puzzle. Cell. 109(Suppl.):S81-S96.

20. Hayden, M.S., and Ghosh, S. 2004. Signaling to NF-kappaB. Genes Dev. 18:2195-2224.

21. Krappmann, D., and Scheidereit, C. 2005. A pervasive role of ubiquitin conjugation in activation and termination of IkappaB kinase pathways. EMBO Rep. 6:321-326.

22. Sanjabi, S., Hoffmann, A., Liou, H.C., Baltimore, D., and Smale, S.T. 2000. Selective requirement for c-Rel during IL-12 P40 gene induction in macrophages. Proc. Natl. Acad. Sci. U. S. A. 97:12705-12710.

23. Grumont, R., et al. 2001. c-Rel regulates interleukin 12 p70 expression in CD8(+) dendritic cells by specifically inducing p35 gene transcription. J. Exp. Med. 194:1021-1032.

24. Traenckner, E.B., Wilk, S., and Baeuerle, P.A. 1994. A proteasome inhibitor prevents activation of NF-kappa B and stabilizes a newly phosphorylated form of I kappa B-alpha that is still bound to NF-kappa B. ЕMBOJ. 13:5433-5441.

25. Palombella, V.J., Rando, O.J., Goldberg, A.L., and Maniatis, T. 1994. The ubiquitin-proteasome pathway is required for processing the NF-kappa B1 
precursor protein and the activation of NF-kappa B. Cell. 78:773-785.

26. Alkalay, I., et al. 1995. Stimulation-dependent I kappa B alpha phosphorylation marks the NF-kappa B inhibitor for degradation via the ubiquitin-proteasome pathway. Proc. Natl. Acad. Sci.U.S. A. 92:10599-10603.

27. Groll, M., et al. 1997. Structure of 20S proteasome from yeast at 2.4 A resolution. Nature. 386:463-471.

28. Gaczynska, M., Rock, K.L., and Goldberg, A.L. 1993. Gamma-interferon and expression of MHC genes regulate peptide hydrolysis by proteasomes. Nature. 365:264-267.

29. Driscoll, J., Brown, M.G., Finley, D., and Monaco, J.J. 1993. MHC-linked LMP gene products specifically alter peptidase activities of the proteasome. Nature. 365:262-264.

30. Boes, B., et al. 1994. Interferon gamma stimulation modulates the proteolytic activity and cleavage site preference of 20S mouse proteasomes. J. Exp. Med. 179:901-909.

31. Nandi, D., Jiang, H., and Monaco, J.J. 1996. Identification of MECL-1 (LMP-10) as the third IFN- gamma-inducible proteasome subunit. J. Immunol. 156:2361-2364.

32. Kloetzel, P.M. 2004. The proteasome and MHC class I antigen processing. Biochim. Biophys. Acta. 1695:225-233.

33. Kuckelkorn, U., et al. 2002. Link between organspecific antigen processing by $20 \mathrm{~S}$ proteasomes and CD8(+) T cell-mediated autoimmunity. J. Exp. Med. 195:983-990.

34. Groll, M., and Huber, R. 2004. Inhibitors of the eukaryotic $20 \mathrm{~S}$ proteasome core particle: a structural approach. Biochim. Biophys. Acta. 1695:33-44.

35. Alvarez-Castelao, B., and Castano, J.G. 2005. Mechanism of direct degradation of IkappaBalpha by 20S proteasome. FEBS Lett. 579:4797-4802.

36. Petrof, E.O., et al. 2004. Probiotics inhibit nuclear factor-kappaB and induce heat shock proteins in colonic epithelial cells through proteasome inhibition. Gastroenterology. 127:1474-1487.

37. Griffin, T.A., et al. 1998. Immunoproteasome assembly: cooperative incorporation of interferon gamma (IFN-gamma)-inducible subunits. J. Exp. Med. 187:97-104.
38. Fuss, I.J., et al. 2006. Both IL-12p70 and IL-23 are synthesized during active Crohn's disease and are down-regulated by treatment with anti-IL-12 p40 monoclonal antibody. Inflamm. Bowel Dis. 12:9-15.

39. Present, D.H., et al. 1999. Infliximab for the treatment of fistulas in patients with Crohn's disease. N. Engl. J. Med. 340:1398-1405.

40. Monteleone, G., et al. 2004. A failure of transforming growth factor-beta 1 negative regulation maintains sustained NF-kappaB activation in gut inflammation. J. Biol. Chem. 279:3925-3932.

41. Monteleone, G., et al. 2005. Post-transcriptional regulation of Smad7 in the gut of patients with inflammatory bowel disease. Gastroenterology. 129:1420-1429.

42. Fichtner-Feigl, S., Fuss, I.J., Preiss, J.C., Strober, W., and Kitani, A. 2005. Treatment of murine Th1and Th2-mediated inflammatory bowel disease with NF-кB decoy oligonucleotides. J. Clin. Invest. 115:3057-3071. doi:10.1172/JCI24792.

43. Cerundolo, V., et al. 1990. Presentation of viral antigen controlled by a gene in the major histocompatibility complex. Nature. 345:449-452. 